\title{
X-ray He-like ion diagnostics: New computations for photoionized plasmas
}

\section{Influence of different parameters}

\author{
O. Godet ${ }^{1}$, S. Collin' ${ }^{2}$, and A.-M. Dumont ${ }^{2}$ \\ 1 CESR, 9 av. du Colonel Roche, 31028 Toulouse Cedex 4, France \\ e-mail: 0livier.Godet@cesr.fr \\ ${ }^{2}$ LUTH, Observatoire de Paris, Section de Meudon, Place Jules Janssen, 92195 Meudon Cedex, France
}

Received 5 March 2004 / Accepted 29 June 2004

\begin{abstract}
In a previous article, with a new code designed for a photoionized thick hot medium using a full transfer treatment of both lines and continuum, we have studied the He-like emission of a medium photoionized by an X-ray source. We considered the influence of density, ionization parameter and column density. We stressed that photoionized media are not uniform in temperature and we showed that photoexcitation and large column densities should be considered to avoid misinterpretation of the properties of the medium. To complete the possible situations observed in photoionized plasmas, we consider here the influence of microturbulence due for instance to a velocity gradient, of different element abundances, and of different ionizing spectral distributions. All new situations are computed using the same code, but with an improved version of our He-like atomic model. We give values of the $G$ and $R$ ratios, the equivalent width, and the ionic column densities with a greater accuracy than previously achieved. A large microturbulence results in a decrease of the $G$ ratios for large column densities, for which the He-triplet resonant lines are optically thick. On the contrary, the $R$ ratio is almost independent of the dispersion velocity and the element abundances, as long as the intercombination lines are optically thin. Through two "universal diagrams" not depending on any parameter, we also present a way to determine the ionization parameter and the total column density of the emitting regions from the temperature of the observed radiative recombination continuum of a given ion and its column density.
\end{abstract}

Key words. plasmas - X-rays: galaxies - galaxies: active

\section{Introduction}

In the era of the new X-ray space observatories XMM-Newton and Chandra, the complexity of the soft X-ray spectra of several objects has been discovered. They exhibit tens of emission lines, in particular the He-like ion lines of the $n=2$ complex, which can be used as potential diagnostics to probe the physical state and to constraint the geometry of the emitting regions.

It is well known that in collisional media, the He-like ion line ratios $R$ and $G$ are sensitive respectively to the density and the temperature of the emitting medium (see e.g. Gabriel \& Jordan 1969, 1972, 1973). We recall that these ratios are defined as:

$R=\frac{z}{x+y} \quad G=\frac{z+x+y}{w}$

where $z$ is the forbidden line $\left(1 \mathrm{~s}^{2}{ }^{1} \mathrm{~S}-2 \mathrm{~s}{ }^{3} \mathrm{~S}\right), x$ and $y$ are the intercombination lines $\left(1 \mathrm{~s}^{2}{ }^{1} \mathrm{~S}-2 \mathrm{p}{ }^{3} \mathrm{P}_{1,2}\right)$ and $\mathrm{w}$ is the resonant line $\left(1 \mathrm{~s}^{2}{ }^{1} \mathrm{~S}-2 \mathrm{p}{ }^{1} \mathrm{P}\right)$.

More recently, these calculations have begun to be extended to photoionized plasmas, for objects such as active galactic nuclei (AGN) and X-ray binaries (see the references given in Coupé et al. 2004, called hereafter Paper I).

Aware of the strong potential of the He-like diagnostics to probe these objects, we have undertaken an extensive study of photoionized media. In Paper I, we concentrated on the influence of the density $\left(n_{\mathrm{H}}\right)$, the ionization parameter $(\xi)$ and the column density $(C D)$ on the structure of the emitting region and the He-like spectrum. We computed a grid of models, encompassing a large number of cases where X-ray lines are observed. We used our code, "Titan", designed for photoionized thick hot media, which performs a full transfer treatment of both lines and continuum (see below), and takes into account all relevant physical processes in the X-ray range. This previous study led to some important conclusions concerning photoionized media:

- It is difficult to define a single temperature for the line emitting region, because a photoionized medium is not uniform in temperature.

- The $G$ ratios are almost never equal to the pure recombination value. For low values of $C D, G$ is smaller, owing to 
photoexcitation of the resonance line, and for high values of $C D$, it is larger, owing to photon destruction during the process of resonant scattering.

- From the study of a single ion, a given value of the $G$ ratio and a given equivalent width (EW) could exist for a small $C D$ and a small $\xi$, or for a large $C D$ and a large $\xi$. Other features of the spectrum must be used to resolve this uncertainty.

This second paper has two motivations. First, the results mentioned above were obtained with a relatively simple He-like atomic model allowing only a qualitative description of the He-like emitting region within an accuracy of $50 \%$ for the pure recombination case as well as for the pure collisional case. Aware of this problem, we implemented additional levels to build a more elaborate He-like atomic model for the C V, N VI, $\mathrm{O}$ VII and Fe XXV ions and checked our conclusions given in Paper I. Note that the accuracy obtained with the previous atomic model is better than that which could be obtained when using the escape probability approximation: the errors on the line intensities and line ratios reach thus one order of magnitude for thick media (Dumont et al. 2003) and typically a factor two for "moderate" $C D$ (Collin et al. 2004).

Secondly, we complete the study of photoionized media in considering other possible situations: the implications of a microturbulent velocity added to the thermal velocity within the plasma, and the influence of the element abundances on the structure and emission properties with the example of an over/underabundance of oxygen, an important element in X-ray emitting and absorbing media (e.g. the Seyfert 2 nuclei and the Warm Absorbers). Also, in Paper I, we initiated an investigation of the influence of the spectral distribution using an ionizing power-law continuum with various spectral indexes. Aware that a power law is a poor representation of the continuum in AGN in an energy range extending from UV to the $\mathrm{X}$-rays, we decided to continue this work using incident continua closer to the mean quasar spectrum. Laor et al. (1997) have obtained from a compilation of radio quiet quasars a "mean spectrum". We use this continuum from $13.6 \mathrm{eV}$ to $100 \mathrm{keV}$, since we are not interested in the continuum below $13.6 \mathrm{eV}$, which does not contribute to the ionization of heavy elements. If it would extend in the optical and infrared band, it could however contribute appreciably to the heating at high density through free-free process, and to the cooling at high ionization flux through Compton process. It has a spectral in$\operatorname{dex} \alpha=1.77$ from $13.6 \mathrm{eV}$ to $2 \mathrm{keV}$, and $\alpha=1$ above $2 \mathrm{keV}$. We call it the "AGN1" continuum, in reference to the "AGN" continuum defined in Paper I as a simple power law with $\alpha=0.7$ from $13.6 \mathrm{eV}$ to $100 \mathrm{keV}$. However, since it is well known that in many objects the spectral index above $2 \mathrm{keV}$ is close to 0.7 , we also use an "AGN2" continuum with $\alpha=0.7$ above $2 \mathrm{keV}$.

In Paper I we reviewed the different X-ray emitting photoionization media observed presently with XMM-Newton and with Chandra: Seyfert 1-2, X-ray binaries. Here we focus on the pure emission line spectrum seen in reflection as in Seyfert 2, because such a spectrum does not depend on the proportion of continuum hidden by the emission region. To this aim we have given all the results (equivalent widths, line ratios, spectra) as they would be observed in reflection. A consequence is that the EW are much larger than in Paper I, where they were given relative to the incident continuum. Note that the EW are here upper bounds, as some sources of the continuum transmitted or scattered could be only partly seen, even in Seyfert 2 nuclei.

In the next section we review the computational method and give a short description of the atomic model. Sections 3 and 4 are devoted to a discussion of the influence of the various parameters on the structure and the emission of the medium, and Sect. 5 summarizes the results.

\section{The computational method}

This work is performed with our stationary photoionization code Titan used in Paper I (for a detailed description of the code, see Dumont et al. 2000, 2003). Below, we give a short description of the code and its originality with respect to the other photoionization codes.

Titan uses the Accelerated Lambda Iteration (ALI) method to solve the line and the continuum transfer. It is unprecedented from the point of view of the line transfer for photoionization codes and allows us to compute very precisely the line intensities, even for the most optically thick lines. This cannot be performed with the other photoionization codes like XSTAR (Kallman \& Krolik 1995) and Cloudy (Ferland et al. 1998), which use an integral method, the so-called "escape probability" approximation, instead of solving the line transfer and sometimes even the continuum transfer. With the new data obtained with XMM-Newton and Chandra, it is crucial to use a full transfer method as in Titan to properly compute the spectrum in conditions appropriate for the X-ray emission regions of AGN. Indeed, it was shown in Dumont et al. (2003) and in Collin et al. (2004) that the line intensities, mainly the resonant line, cannot be computed properly with the "escape probability" approximation, owing to the "photon destruction" process due to photoionization by the line photon of ions having lower ionization potential for large $C D$, and to a bad estimation of the photoexcitation rate of resonance lines by the incident continuum for moderate $C D$.

A plane parallel slab of gas is irradiated by an incident spectrum, whose frequency integrated flux is equal to $F$. We define the "ionization parameter" $\xi=4 \pi F / n_{\mathrm{H}} \mathrm{erg} \mathrm{cm} \mathrm{s}{ }^{-1}$, where $n_{\mathrm{H}}$ is the hydrogen number density in the plasma. The gas composition includes the 10 most abundant elements $(\mathrm{H}, \mathrm{He}, \mathrm{C}, \mathrm{N}, \mathrm{O}$, $\mathrm{Ne}, \mathrm{Mg}, \mathrm{Si}, \mathrm{S}, \mathrm{Fe}$ ) and all their ionic species, i.e. 102 ions. A multi-level description is included up to $n=5$ for $\mathrm{H}$-like and Li-like ions, $n=4$ for He-like ions and $n=3$ for O IV and O V, where $n$ is the principal quantum number. Interlocking between excited levels is included. Other ions are treated more approximatively.

Titan includes all relevant physical processes from each level, notably photoionization and photoexcitation, which is very important to avoid misinterpretation of the He-like diagnostics, and all induced processes. The populations of each level are computed solving the set of ionization equations coupled with the set of statistical equations describing the excitation equilibrium, taking into account radiative and collisional 
ionizations in all levels and recombination in all levels, and radiative and collisional excitations and deexcitations for all transitions. The energy balance is ensured locally with a precision of $0.01 \%$, and globally with a precision of $1 \%$.

\subsection{The new He-like atom model}

In Paper I, we used a model for He-like ions with only 11 levels and a continuum. Then we implemented additional levels for the most important He-like ions (C V, N VI, O VII and Fe XXV). The sources of the data are given in Paper I, so we do not recall them here. The new atomic model includes 15 levels and a continuum to take into account all the terms for $n=2$ and $n=3$, rather than a single superlevel as was previously done for $n=3$, and 2 superlevels gathering the singlet and triplet levels for $n=4$. Recombination on the upper levels, $n>4$, are taken into account and are distributed among the lower levels according to their statistical weights. They represent at most $50 \%$ of the total recombination rates in all our computations. Satellite lines are not included but they should be negligible, like the dielectronic recombinations, in the range of temperature where the important ions are present for the media considered here (for instance $10^{5} \mathrm{~K}$ for OVII, and $10^{6} \mathrm{~K}$ for Fe XXV).

\subsection{Models}

We ran models with a range of densities from $10^{7}$ to $10^{12} \mathrm{~cm}^{-3}$, a range of column densities from $10^{18} \mathrm{~cm}^{-2}$ to $3 \times 10^{23} \mathrm{~cm}^{-2}$ and a range of ionization parameters from 30 to 1000, with the new atomic data. We assume cosmic abundances with respect to hydrogen (Allen 1973): He: $0.085 ; \mathrm{C}: 3.3 \times 10^{-4}$; N: $9.1 \times 10^{-5}$; O: $6.6 \times 10^{-4}$; Ne: $8.3 \times 10^{-5} ; \mathrm{Mg}: 2.5 \times 10^{-5}$; Si: $3.3 \times 10^{-5}$; S: $1.6 \times 10^{-4}$; Fe: $3.2 \times 10^{-5}$. We also computed models with an oxygen abundance half, twice and six times the cosmic abundance. The latter is an "extreme" value, certainly too high if we consider that the oxygen abundance is fixed by stellar nucleosynthesis. We also take into account the possible existence of a microturbulent velocity (cf. Appendix for a detailed explanation of what we call microturbulence) added to the thermal velocity. We choose two values: $V_{\mathrm{d}}=$ 300 and $V_{\mathrm{d}}=3000 \mathrm{~km} \mathrm{~s}^{-1}$. The former is representative of the dispersion velocity found in the X-ray spectrum of several Seyfert 1 and 2 galaxies (see e.g. Ogle et al. 2003, and Kinkhabwala et al. 2002 for NGC 1068). The latter is an "extreme" value appropriate for the broad absorption line region (BAL). In Paper I, the incident spectrum was a power law, $F_{v} \propto v^{-1}$, extending from $0.1 \mathrm{eV}$ to $100 \mathrm{keV}$. We will call it the "standard continuum" (SC). This being a poor representation of the continuum in AGN, we also use the AGN1 and AGN2 continua as mentioned earlier. We deal only with a constant density inside the slab.

\section{Results}

To show the different effects properly, we consider them separately.

\subsection{New atomic data}

First, it is interesting to consider whether the new atomic model for C V, N VI, O VII, and Fe XXV ions has a significant impact on the thermal and ionic stratification of the medium. An important point to bear in mind is that the location of the He-like emitting region is closely linked to the stratification of the medium, as stressed in Paper I. Indeed, we showed that a photoionized medium is divided into several zones according to its thickness: a "hot skin" located below the surface for which the temperature is roughly constant and the elements are highly ionized, an "intermediate" zone where the temperature decreases and the elements are less ionized, and a "cold" zone where the elements are in low ionization states. In Fig. 1, we show an example of the temperature and fractional ionic abundances of the O VII and O VIII ions versus the column density computed from the surface to the local point $C D(z)$ for a medium with $C D=10^{23} \mathrm{~cm}^{-2}, n_{\mathrm{H}}=10^{7} \mathrm{~cm}^{-3}$ and $\xi=100$. It is appropriate for the Warm Absorber in Seyfert 1 galaxies and the X-ray emission region in Seyfert 2 galaxies. For this moderate value of $C D$, only the "hot" and "intermediate" zones could be seen. It is in this "intermediate" zone, inhomogeneous in temperature and close to the back side of the slab, that the O VII spectrum is formed. A comparison with Fig. 2 of Paper I shows that the new atomic model of He-like ions almost does not modify the fractional ionic abundance, and the new equilibrium in temperature is globally close to the previous one with a relative discrepancy of $10 \%$.

In Fig. 2, several examples of the temperature $T_{\max }$ of the $\mathrm{O}$ VIII ions are given as a function of the column density of the $\mathrm{O}$ VII ions, according to some given $\xi$-values (panel B), and to some given values of the total column density $C D$ (panel $\mathrm{A}$ ). $T_{\max }$ is the maximum electron temperature in the region where the O VIII ions dominate. We find that the curves with the new and old atomic data are quite similar. As stressed in Paper I, this figure is important, since it relates two quantities $T_{\max }$ (O VIII) and $C D(\mathrm{O}$ VII $)$ which can be deduced directly from the observed spectra, $T_{\max }(\mathrm{O}$ VIII) corresponding to an upper bound of the temperature derived from the $\mathrm{O}$ VII radiative recombination continuum (RRC).

The new He-like model allows us to get a better accuracy on the ratios $R$ and $G$. Our previous model for the He-like ions gave $R$ correct to only $20 \%$. We find a $G$-value in the pure recombination case for a thin slab $(G=5.6)$ for O VII ions similar to the value given in Kinkhabwala et al. (2002), and a $R$-value for O VII ions $\left(R=3.9\right.$ for $n_{\mathrm{H}}=10^{7} \mathrm{~cm}^{-3}$ and $\xi=100$ i.e. for a temperature $T \sim 10^{5} \mathrm{~K}$ ) similar to Porquet $\&$ Dubau (2000) in the case of a very thin medium $\left(C D=10^{18} \mathrm{~cm}^{-2}\right)$.

Figure 3 shows the $G$ ratios versus $C D$ for models with a range of $\xi$ from 30 to 300 and $n_{\mathrm{H}}=10^{7} \mathrm{~cm}^{-3}$, and a range of $C D$ from $10^{18}$ to $3 \times 10^{23} \mathrm{~cm}^{-2}$. The new $G$-values fit well the previous $G(5 \%)$ for all ions except for OVII at large $C D$ at $\xi \leq 30$. In this figure, the long dashed line at $\xi=100$ symbolizes the saturation of the $G$ ratio for the $\mathrm{C} \mathrm{V}$ and $\mathrm{Fe} X X V$ ions. Note that $G(\mathrm{O}$ VII) saturates for smaller $C D$ at $\xi=30$. We recall that this saturation is due to the limit of the $C D_{\text {ion }}$ according to the values of $\xi$, as shown in Fig. 4 in Paper I. 

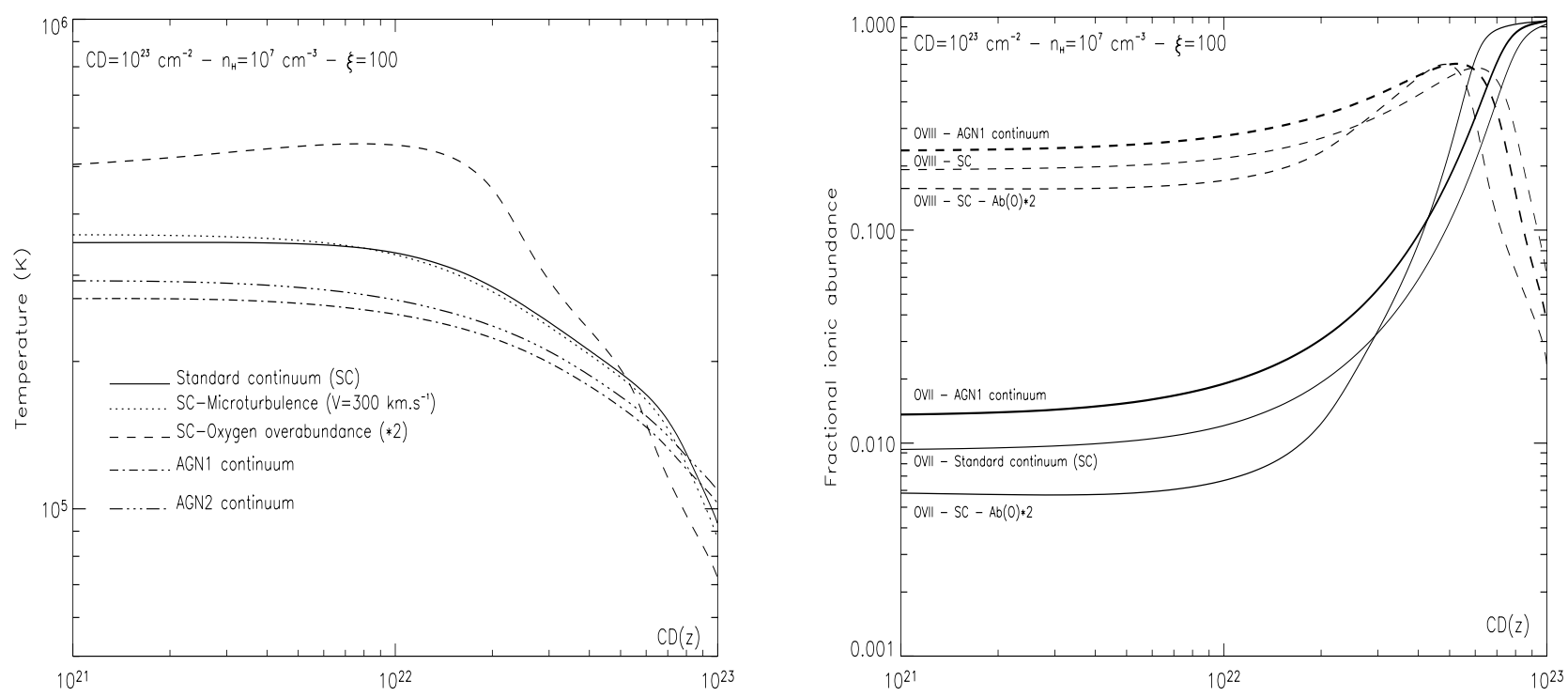

Fig. 1. Left panel: temperatures versus the column density $C D(z)$ for $C D=10^{23} \mathrm{~cm}^{-2}, n_{\mathrm{H}}=10^{7} \mathrm{~cm}^{-3}$ and $\xi=100$. These temperatures are computed for several situations: the new atomic data (solid line); microturbulence $V_{\mathrm{d}}=300 \mathrm{~km} \mathrm{~s}^{-1}$ (dotted line); twice the oxygen cosmic abundance (dashed line); irradiation by the AGN1 and AGN2 continua (dot-dashed line and 3 dot-dashed line respectively), the other ones being computed with the standard continuum (SC). Right panel: fractional ionic abundances of the O VII and O VIII ions versus $C D(z)$ for the same model as mentioned above: twice the oxygen cosmic abundance; irradiation by the AGN1 continuum. The labels on the curves give the corresponding situations considered.
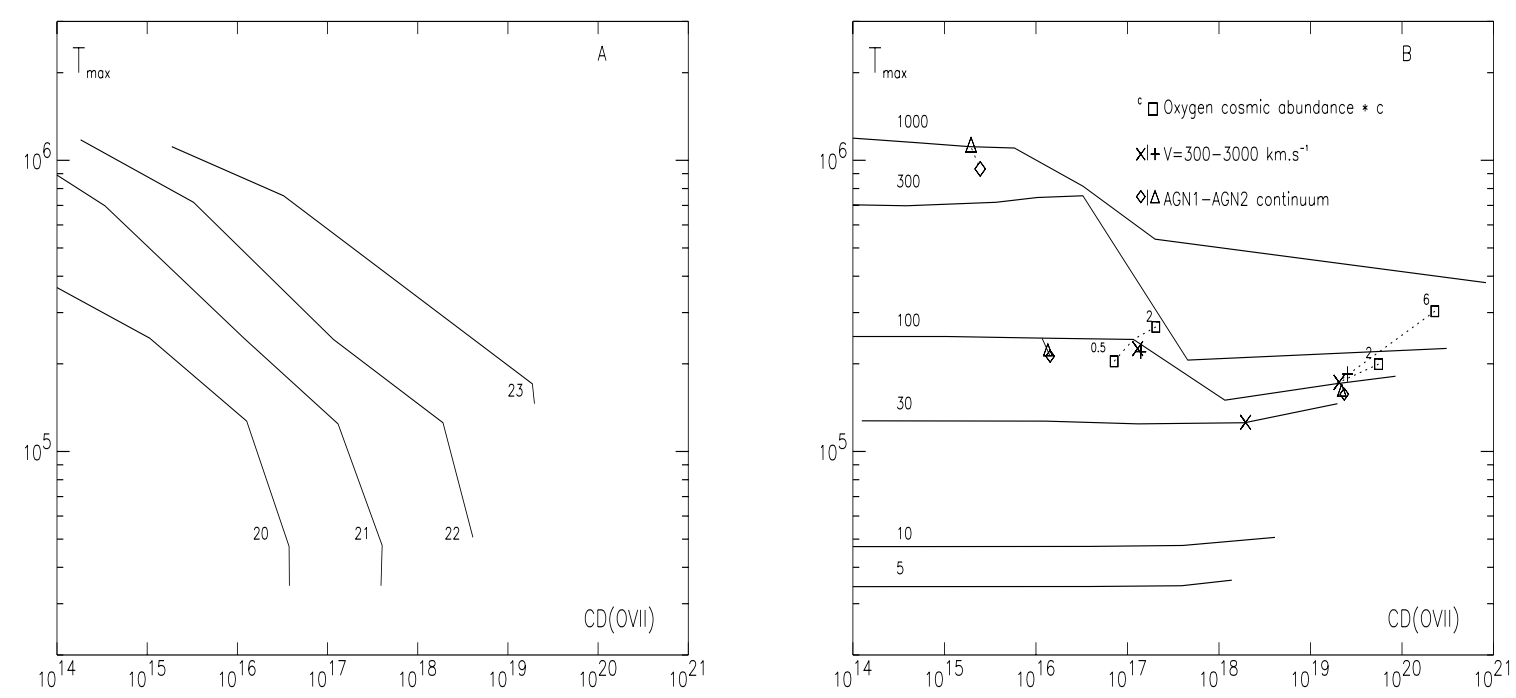

Fig. 2. Maximum temperatures $T_{\max }(\mathrm{K})$ of the regions where $\mathrm{O}$ VIII dominate as a function of the O VII column density $\left(\mathrm{cm}^{-2}\right)$ according to: A) some given $C D$-values labelled on the curves in logarithm; B) some given $\xi$-values labelled on the curves: the symbol "+" and the crosses for $V_{\mathrm{d}}=3000-300 \mathrm{~km} \mathrm{~s}^{-1}$ respectively; the squares for the models with a varying oxygen abundance; the diamonds and the triangles for the AGN1 and AGN2 continua. The dotted lines in the right figure link models having the same value of $\xi$ and $C D$.

Slightly more significant discrepancies between the previous and new $R$-values can be seen in Fig. 4, showing $R$ for the O VII ion versus the density $n_{\mathrm{H}}$ for $C D=10^{21} \mathrm{~cm}^{-2}$ and $\xi=100$. At low densities, $R(\mathrm{O}$ VII) computed with the new He-like model is larger than the previous one by about $20 \%$, while at high densities, the new $R$-values fit well the previous $R$-values. This is because at high densities, the $n=2$ intercombination and forbidden line transitions are basically driven by collisions. It results in an increased forbidden line and a decreased intercombination line. Note that a similar behaviour is also seen for the new $R(\mathrm{C} \mathrm{V}, \mathrm{N} \mathrm{VI})$-values, while the new
$R(\mathrm{Fe} \mathrm{XXV})$-values present small differences with respect to the previous values $( \pm 2 \%)$.

In Fig. 5, we also give the EW computed as the sum of the O VII triplet lines with respect to the reflected continuum.

\subsection{Influence of microturbulence}

We take into account here the existence of microturbulence which, unlike macroturbulence (like in the BLR of AGN), intervenes in the line transfer, as explained earlier. 

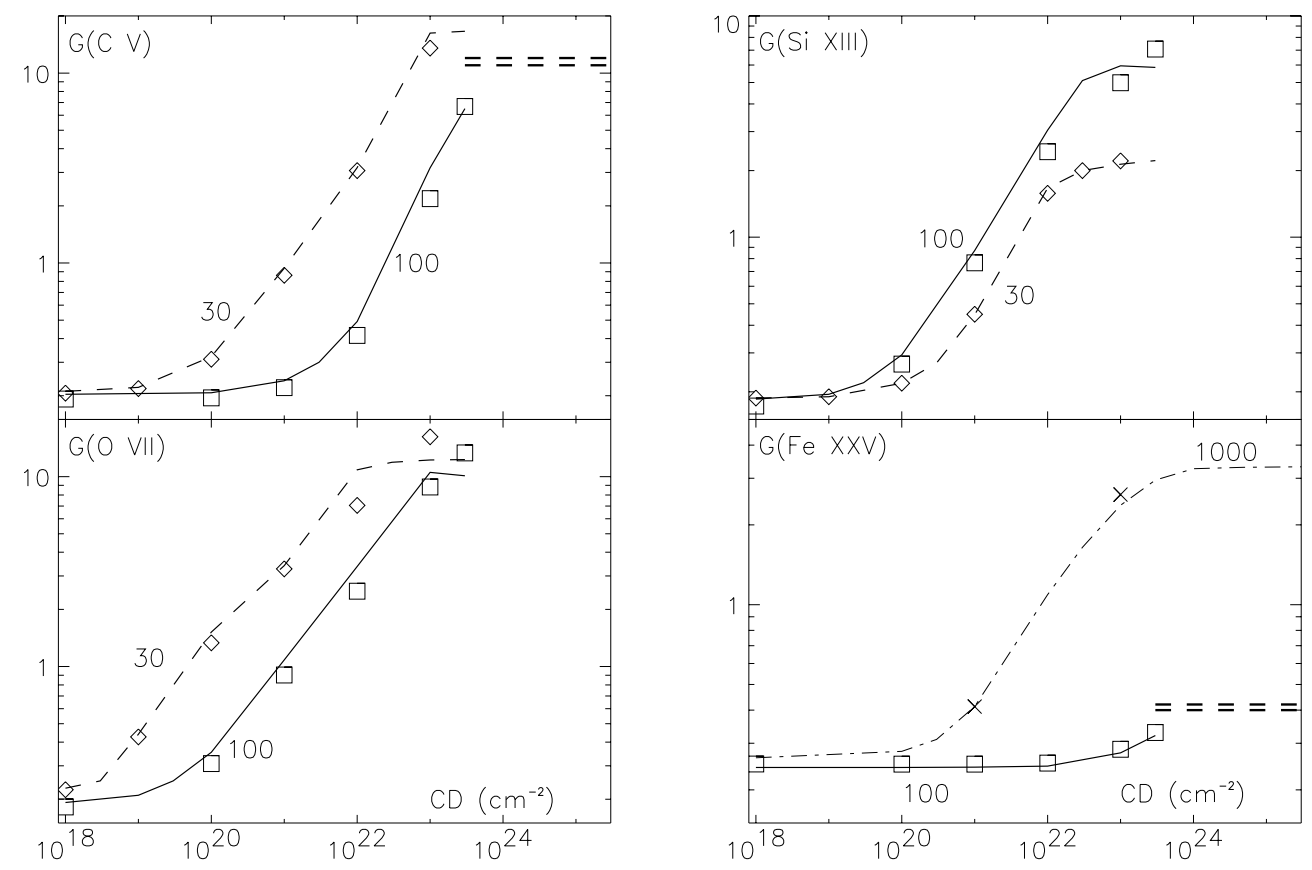

Fig. 3. $G$ ratios in the reflected spectrum versus $C D$ : Comparison between the new (the solid line: $\xi=100$; dashed line: $\xi=30$ ) and the previous (squares: $\xi=100$; diamonds: $\xi=30$ ) atomic data for media with $n_{\mathrm{H}}=10^{7} \mathrm{~cm}^{-2}$ irradiated by the standard continuum. For the Fe XXV ions and the curve labelled at $\xi=1000$, the previous (dot-dashed line) and new (crosses) atomic data are shown. The double long dashed line for the $\mathrm{C} \mathrm{V}$ and Fe XXV ions symbolizes the saturation of the $G$ ratio above a certain value of $C D$.

First, it is interesting to ask whether a microturbulent velocity has a significant influence on the plasma temperature and on the line intensities. Microturbulence decreases the optical thickness of the lines at the line center, $\tau_{0}$, as it is inversely proportional to $V_{\mathrm{d}}$. There is consequently an increase of emissivity of the thick lines, as the rate of incident radiative excitation for these lines increases when $\tau_{0}$ decreases, while the thin lines (for instance the forbidden lines) are almost not affected, as stressed in Paper I. Thus, the resonant lines w being optically thick for the "moderate" and large $C D$, their intensities should increase when $\tau_{0}$ decreases, while for the thin media where even the thickest lines are optically thin, the line intensities do not vary strongly. This is well illustrated in Fig. 6 (panel B) which shows the reflected spectra of models computed with the standard continuum, $C D=10^{23} \mathrm{~cm}^{-2}, \xi=100, n_{\mathrm{H}}=10^{7} \mathrm{~cm}^{-3}$, when comparing the panel B with $V_{\mathrm{d}}=300 \mathrm{~km} \mathrm{~s}^{-1}$ to the panel A without microturbulence. All spectra are convolved with a Gaussian profile of a $F W H M$ equal to $1000 \mathrm{~km} \mathrm{~s}^{-1}$ (we recall that the lines can also be broadened by macroturbulence). As shown below, it will have also some consequences on the $G$ ratios.

Microturbulence has a small influence on the temperature. For a "moderate" medium with $C D=10^{23} \mathrm{~cm}^{-2}$, the temperature computed for $V_{\mathrm{d}}=300 \mathrm{~km} \mathrm{~s}^{-1}$ is very similar to that computed without microturbulence (cf. Fig. 1). For $V_{\mathrm{d}}=$ $3000 \mathrm{~km} \mathrm{~s}^{-1}$, it increases by less than $15 \%$ at the illuminated surface and decreases by less than $30 \%$ at the back side with respect to that computed without microturbulence.

The ionic fractional abundances do not vary strongly when compared to the previous values, notably for O VII and $\mathrm{O}$ VIII ions. It implies that the ionic column densities are close to those found without microturbulence, and thus the

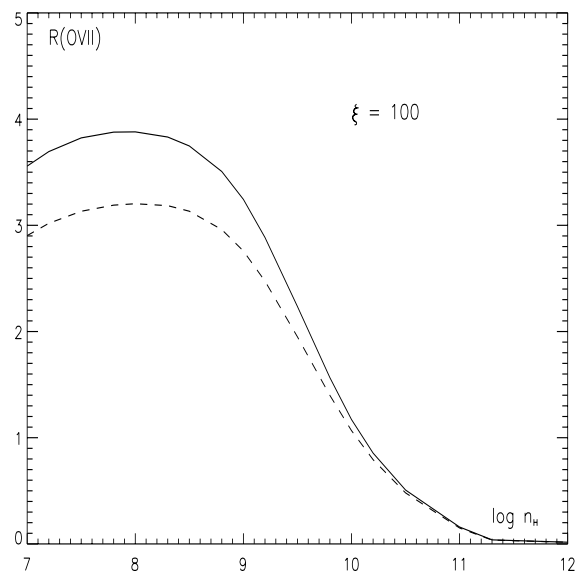

Fig. 4. $R$ ratio for the $\mathrm{O}$ VII ion in the reflected spectrum versus the density $n_{\mathrm{H}}$ for media with $C D=10^{21} \mathrm{~cm}^{-2}, \xi=100$, photoionized by the standard continuum. The thick solid (dashed) lines correspond to the $R$-values computed with the new (old) atomic He-like model.

$T(\mathrm{O} \mathrm{VIII}) / C D(\mathrm{O} \mathrm{VII})$ diagrams in Fig. 2 are almost independent of the microturbulent velocity.

Figure 7 shows the influence of the microturbulent velocity on the $G$ ratios for the O VII ions with $n_{\mathrm{H}}=10^{7} \mathrm{~cm}^{-3}$ and $\xi=100 . G$ decreases when $V_{\mathrm{d}}$ increases for a given $C D$, except for $C D \leq 10^{18} \mathrm{~cm}^{-2}$ for the reasons explained above. Note that $G$ saturates at the same column density with and without microturbulence, but at smaller and smaller values, as $V_{\mathrm{d}}$ increases. Therefore, for the same $G$-value, microturbulence implies a larger CD than that derived from models without microturbulence. Indeed, taking the example of NGC 1068 given in Paper I where $G(\mathrm{O}$ VII) $\sim 2.5$ (see Kinkhabwala et al. 2002), this 

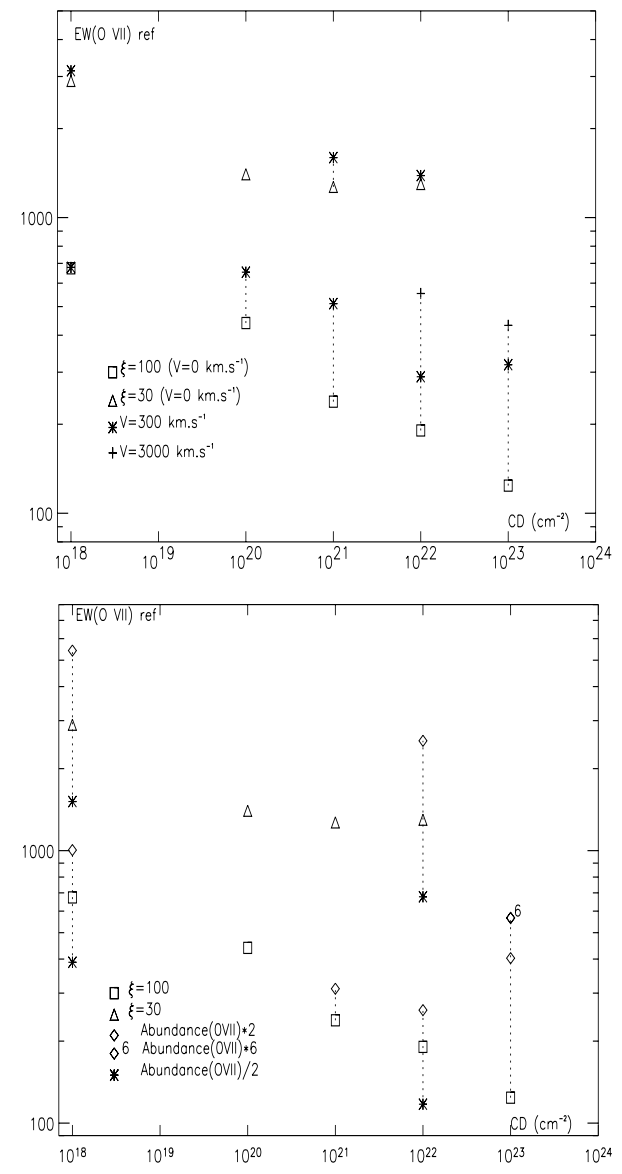

Fig. 5. Reflected EWs of the sum of the O VII triplet versus the column density of the slab. Top panel: models with $V_{\mathrm{d}}=300 \mathrm{~km} \mathrm{~s}^{-1}$ (stars) and $V_{\mathrm{d}}=3000 \mathrm{~km} \mathrm{~s}^{-1}$ (crosses). Bottom panel: models with a variation of the oxygen abundance: abundance increased by a factor of 2 (diamonds). The diamond indexed by " 6 " corresponds to an abundance increased by a factor of 6 for $\xi=100$. The stars correspond to an abundance decreased by a factor of 2 . For the two figures, the squares and the triangles corresponds respectively to the reflected EWs for $\xi=100$ and 30 . The dotted lines on the two figures link models having the same value of $\xi$.

value could be explained with $\xi \sim 100$ and $C D \sim 10^{22} \mathrm{~cm}^{-2}$, in the absence of microturbulence. For $V_{\mathrm{d}}=300 \mathrm{~km} \mathrm{~s}^{-1}$, we find that this same value can be explained with $\xi \sim 100$ and a larger $C D \sim 10^{23} \mathrm{~cm}^{-2}$.

The results are however not trivial, as they depend critically on the transfer treatment at moderate and low $C D$. Since, for small $\xi(\xi \leq 10)$ and small $C D\left(C D=10^{18-19} \mathrm{~cm}^{-2}\right)$, the optical thickness of the $C V$ and $O$ VII resonant lines is still greater than unity, microturbulence has an influence. For instance, for a medium with $C D=10^{18} \mathrm{~cm}^{-2}, n_{\mathrm{H}}=10^{7} \mathrm{~cm}^{-2}, \xi=5$, and $V_{\mathrm{d}}=300 \mathrm{~km} \mathrm{~s}^{-1}$, the $G$-values for these ions decrease by $\sim 40 \%$ with respect to those computed without $V_{\mathrm{d}}$.

The $R\left(\mathrm{O}\right.$ VII)-values with $V_{\mathrm{d}}=300 \mathrm{~km} \mathrm{~s}^{-1}$ are similar to those found without microturbulent velocity, because $R$ is, by definition, the ratio of the forbidden lines over the intercombination lines, which are optically thin. However, for the "moderate" and thick media it can happen that for some $\xi$-values the intercombination line $(x)$ of some ions is no longer optically
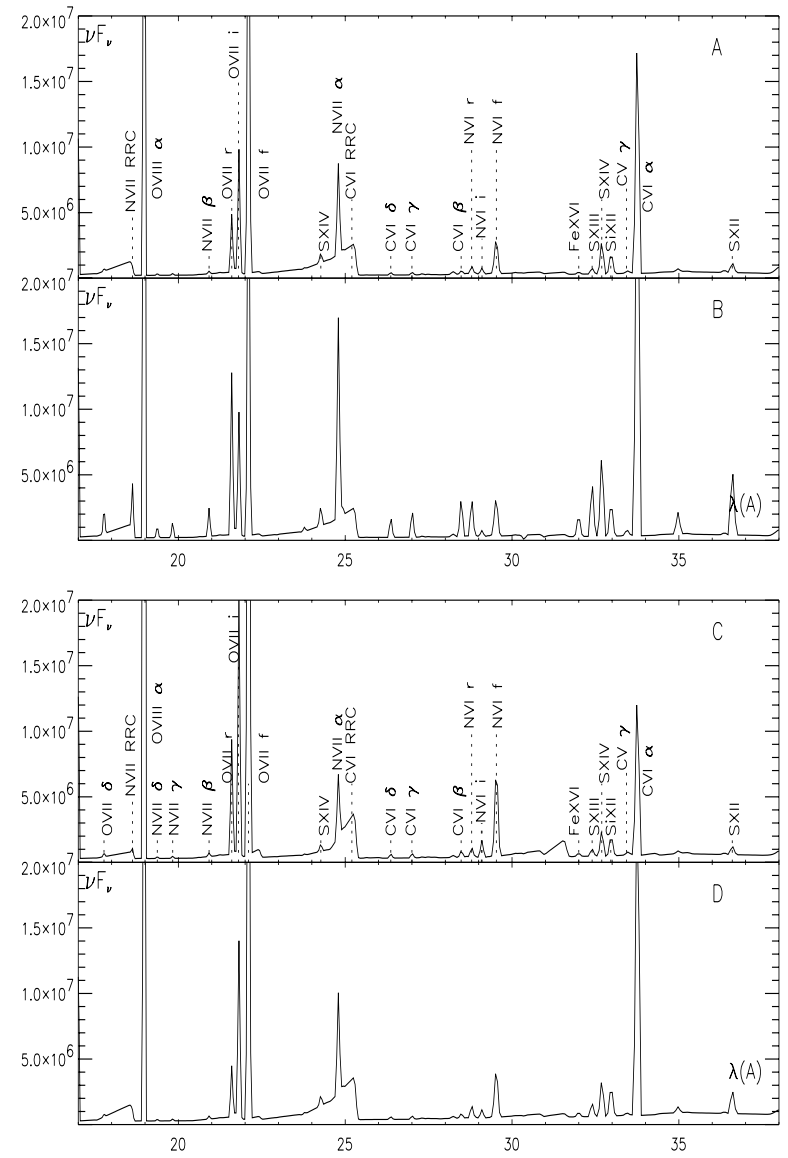

Fig. 6. Examples of reflected spectra given in a part of the RGS energy range for $C D=10^{23} \mathrm{~cm}^{-2}, n_{\mathrm{H}}=10^{7} \mathrm{~cm}^{-3}, \xi=100$ photoionized by the SC continuum. A) No microturbulent velocity $V_{\mathrm{d}}$ and cosmic abundances; B) $V_{\mathrm{d}}=300 \mathrm{~km} \mathrm{~s}^{-1}$ and cosmic abundances; C) no microturbulent velocity and an oxygen abundance six times the cosmic abundance; D) no microturbulent velocity and cosmic abundances, but irradiated with the AGN1 continuum. The label "i" corresponds to the sum of the two intercombination lines $x$ and $y$. All the spectra are convolved with a Gaussian profile of a FWHM equal to $1000 \mathrm{~km} \mathrm{~s}^{-1}$.

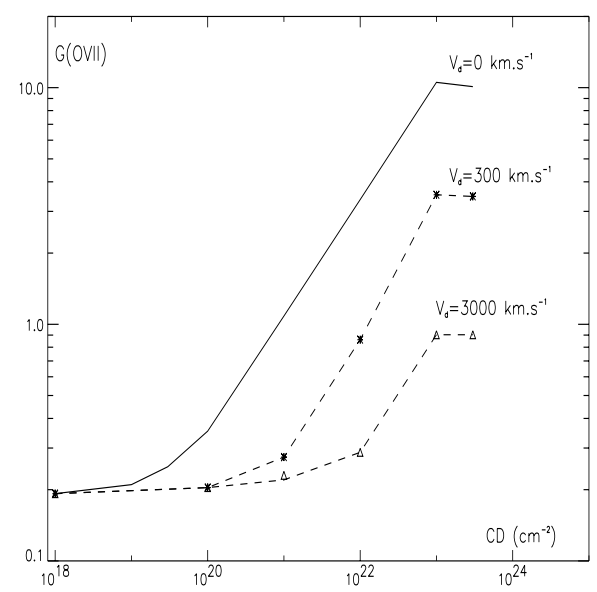

Fig. 7. $G$ ratio in the reflected spectrum for the $\mathrm{O}$ VII triplet versus $C D$ for a medium with $n_{\mathrm{H}}=10^{7} \mathrm{~cm}^{-3}, \xi=100$, photoionized by the standard continuum. The solid line with squares represents models without microturbulence; the dashed line with stars and the dashed line with triangles represent models with a microturbulent velocity of 300 and $3000 \mathrm{~km} \mathrm{~s}^{-1}$ respectively. 
thin, the $y$ and $z$ lines being still optically thin, and $R$ then depends on the microturbulent velocity.

Figure 5 (top panel) also shows the influence of microturbulence, displaying the reflected EW of the sum of the O VII triplet.

\subsection{Oxygen overabundance and underabundance}

We limit ourselves to varying the abundance of the oxygen atom only, as it is an important element in the spectrum of Warm Absorbers-Emitters of Seyfert 1 nuclei and of the X-ray emission region of Seyfert 2 nuclei. We recall that we consider an oxygen abundance half, twice and six times the cosmic abundance.

Unlike microturbulence, varying the oxygen abundance induces a larger variation in the temperature and the fractional abundances, as displayed in Fig. 1 for $C D=10^{23} \mathrm{~cm}^{-2}, n_{\mathrm{H}}=$ $10^{7} \mathrm{~cm}^{-3}, \xi=100$, and an oxygen abundance twice the cosmic abundance. The temperature at the surface irradiated by the ionizing continuum is larger by $\sim 45 \%$ than that computed with the oxygen cosmic abundance, while the temperature of the back side decreases by $\sim 25 \%$. Our interpretation is that heating near the surface should be due to the direct X-rays absorbed on oxygen as well as to the increased back-scattered radiation due to a larger number of O VII photons emitted by the back side. Cooling is not much affected because the emission in the surface layers is dominated by the lines from heavier elements, whose abundances are not changed. On the contrary, at the back side, the O VII cooling dominates and is increased. Thus, the temperature of the back side decreases strongly, but at the same time, heating near the surface causes an increase in temperature. The modifications of the He-like abundances are larger for the light elements (C, N and O) than for the heavier elements ( $\mathrm{Si}, \mathrm{S}$, and $\mathrm{Fe}$ ), as the light He-like elements are present in the same region as oxygen, while the others are present closer to the surface. For an "extreme" value of six times the oxygen cosmic abundance, the differences are more significant,with an increase of the temperature by a factor of 2.5 at the surface, and a decrease of the temperature by $70 \%$ in the back side of the slab with respect to the values computed with the cosmic abundance. This translates into differences in the line intensities, in particular for the light elements. This is well seen in Fig. 6 (panel C) which displays an example of the reflected spectra for $C D=10^{23} \mathrm{~cm}^{-2}, n_{\mathrm{H}}=10^{7} \mathrm{~cm}^{-3}, \xi=100$ and an "extreme" oxygen abundance six times the cosmic abundance. Note that it is mainly the spectral features of the light species that are modified, the heavy species being almost the same, as shown above. Figure 5 (bottom panel) also shows the impact of the oxygen abundance on the reflected EW of the sum of the O VII triplet.

As seen above in Fig. 2 (panel B) with microturbulence, the points with an oxygen under-/over-abundance do not strongly differ from those obtained with the cosmic abundance, except for the extreme increase of oxygen overabundance, where the discrepancies are more significant (see the full circle in the panel B in Fig. 2). So again this diagram appears to be model independent.
The $R(\mathrm{O}$ VII $)$ values are similar to those computed with the cosmic abundance: the forbidden and intercombination lines being optically thin, their intensities vary in the same way with abundance and $R$ consequently does not depend on the abundance. However, for "moderate" and large $C D$ and for some $\xi$-values, the intercombination line, $x$, is optically thick, and $R$ depends on the abundance. Thus, for a model with $C D=$ $10^{23} \mathrm{~cm}^{-2}, n_{\mathrm{H}}=10^{7} \mathrm{~cm}^{-3}, \xi=100$ and an oxygen abundance six times the cosmic abundance, the $R$-value increases within $30 \%$ of that with the cosmic abundance.

For the $G$ ratio, we see in Fig. 8 that for very thin media $\left(C D=10^{18} \mathrm{~cm}^{-2}\right)$, the $G(\mathrm{O}$ VII $)$ value is close to the value computed with the cosmic abundance, because the resonant line is also optically thin and so $G$ almost does not depend on the abundance. For "moderate" and thick media, where the resonant and intercombination $(x)$ lines are optically thick and the forbidden and intercombination $(y)$ lines are still optically thin, $G$ depends on the abundance in a complex way, as shown in Fig. 8. However, this has only a limited impact on the column density of the medium derived for all elements. On the other hand, for an "extreme" value (six times the oxygen cosmic abundance), the $G$-values for the light ions (C V and N VI) show some significant differences with respect to those found with the cosmic abundance, as seen for the $\mathrm{C} \mathrm{V}$ ion in Fig. 8, whereas for the heavy ions (e.g. Si XIII) and O VII ions, $G$ presents some smaller differences (less than $30 \%$ ).

\subsection{The ionizing spectral distribution}

To compare the influence of the spectral distribution incident on the medium, we normalized the different continua so that they have the same flux at $1 \mathrm{keV}$.

The spectral distribution of the incident continuum has an influence on the temperature and on the ionization state. The left panel in Fig. 1 shows the temperature profile for a model with $C D=10^{23} \mathrm{~cm}^{-2}, n_{\mathrm{H}}=10^{7} \mathrm{~cm}^{-3}$ and $\xi=100$ photoionized by the AGN1 and AGN2 continua. The temperature at the surface is lower than that obtained with the standard continuum, while the temperature near the back side is higher. This is translated into differences in the line intensities as we will see below. In the right panel in Fig. 1, only the fractional abundances of the O VII and O VIII ions obtained with the AGN1 continuum are given, since the ionic stratification is globally close to that obtained with the AGN2 models.

Note that in Fig. 2 the AGN1 and AGN2 points are located relatively close to the points corresponding to the standard continuum. Thus, these diagrams are almost independent of the ionizing spectrum.

The panel D in Fig. 6 shows the reflected spectrum for a model with $C D=10^{23} \mathrm{~cm}^{-2}, n_{\mathrm{H}}=10^{7} \mathrm{~cm}^{-3}$ and $\xi=100$, irradiated by the AGN1 continuum. It is similar to that obtained with the AGN2 continuum, which is not displayed here. Indeed, the temperature does not strongly differ between the AGN1 and AGN2 models (cf. Fig. 1), so the line intensities are similar. Both of these spectra present stronger radiative recombination continua (RRC) for N VII and C VI, and stronger C VI $\alpha$, $\mathrm{N}$ VI $\mathrm{i}$ and $\mathrm{f}$, and $\mathrm{O}$ VII $\mathrm{i}$ and $\mathrm{f}$ lines than the reflected spectrum 

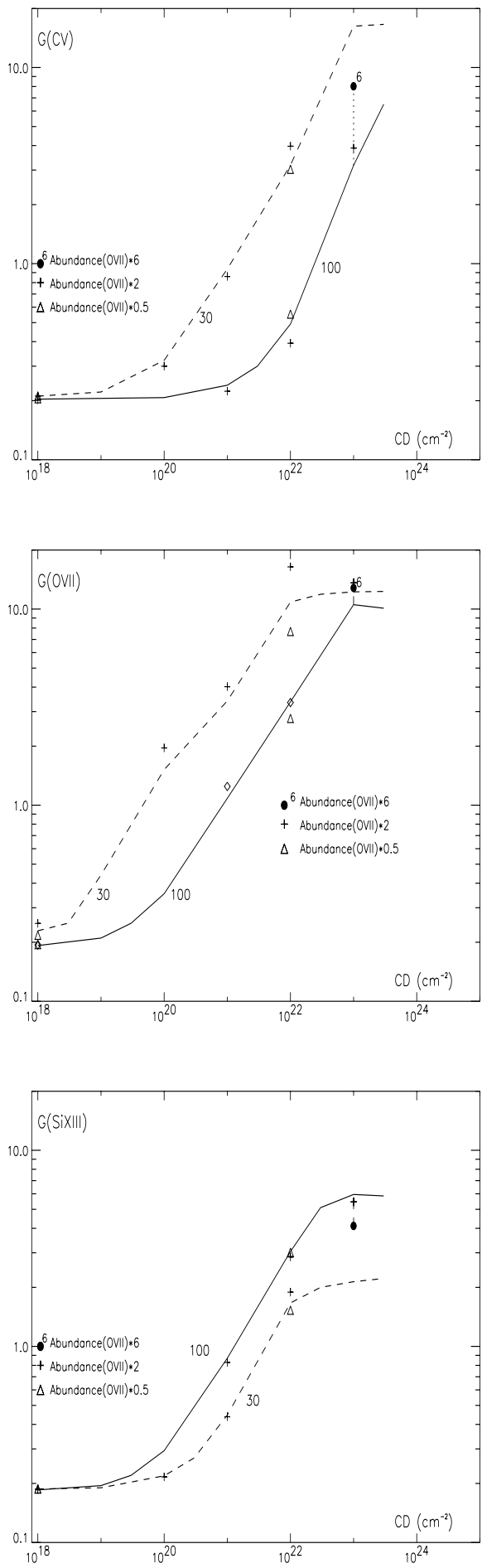

Fig. 8. $G$ ratio in the reflected spectrum for the C V, O VII and Si XIII ions versus the column density for two values of the ionization parameter. The labels on the curves obtained with the oxygen cosmic abundance give the corresponding value of $\xi$. The triangles and the crosses correspond to an abundance 0.5 and 2 times the cosmic abundance for $\xi=30$. The stars and the diamonds correspond to the same for $\xi=100$. The full circle corresponds to an abundance 6 times the cosmic abundance for $\xi=100$.

obtained with the standard continuum (see panel A in Fig. 6). We also give the reflected EW of the sum of the O VII triplet lines computed with the AGN1 and AGN2 continua in Fig. 9.

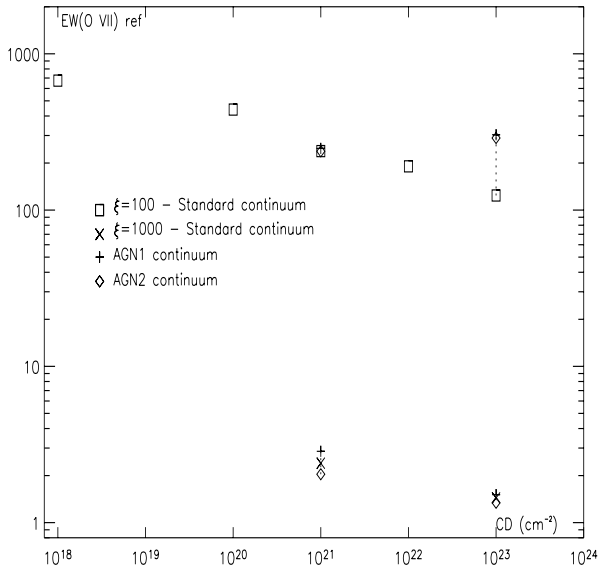

Fig. 9. Same caption as Fig. 5 but for models irradiated by the AGN1 and AGN2 continua both normalized at $1 \mathrm{keV}$ to the standard continuum. The "+" and the diamonds correspond to the AGN1 and AGN2 continua respectively. The squares and the crosses correspond to the values computed with the standard continuum, for $\xi=100$ and $\xi=1000$ respectively.

In Fig. 10, we give $G$ for the O VII and Fe XXV ions computed with the AGN1 and AGN2 continua. Note that for $\xi=1000, G(\mathrm{Fe} X X V)$ presents some discrepancies between the values computed with the AGN1 and AGN2 continua. For $\xi=100$, the $G$-values between the AGN1 and AGN2 continua are relatively similar for all the ions. The $R$ ratio, given in Fig. 11 for the O VII ion, shows differences between the standard and AGN1-2 continua. These differences are significant in the region where $R$ decreases strongly with the density. In the region where $R$ is approximatively constant, the $R$-values are close to those computed with the standard continuum with differences less than $\sim 10 \%$. We observe the same behaviour for the heavy-Z Mg XII, Si XIV and S XV ions. Note also that for all the ions, the $R$-values are very close between the AGN1 and AGN2 continua for the $C D$ and $\xi$ values considered here.

\section{Conclusion}

Using our new version of the photoionization code Titan and improved atomic data for the He-like ions C V$\mathrm{N}$ VI-O VII-Fe XXV, we have computed the line intensities emitted by an X-ray photoionized plasma. We focused on the He-like emission for a range of ionization parameters and column densities, considering several situations met in photoionized objects. The results of this study are:

- The new atomic data of the He-like ions (C V, N VI, $\mathrm{O}$ VII and Fe XXV), taking into account all the separated $n=3$ levels, globally confirms the results obtained with our previous He-like model. We find that the new atomic data lead to $R$ - and $G$-values similar to those computed by other authors in the case of a pure recombination medium. The $R$-values computed with the new atomic data differ by up to $20 \%$ from those previously computed at low densities.

- The different situations considered here show that the diagrams displaying the temperature of the $O$ VIII ion versus the ionic column density of the $O$ VII ions are almost model 

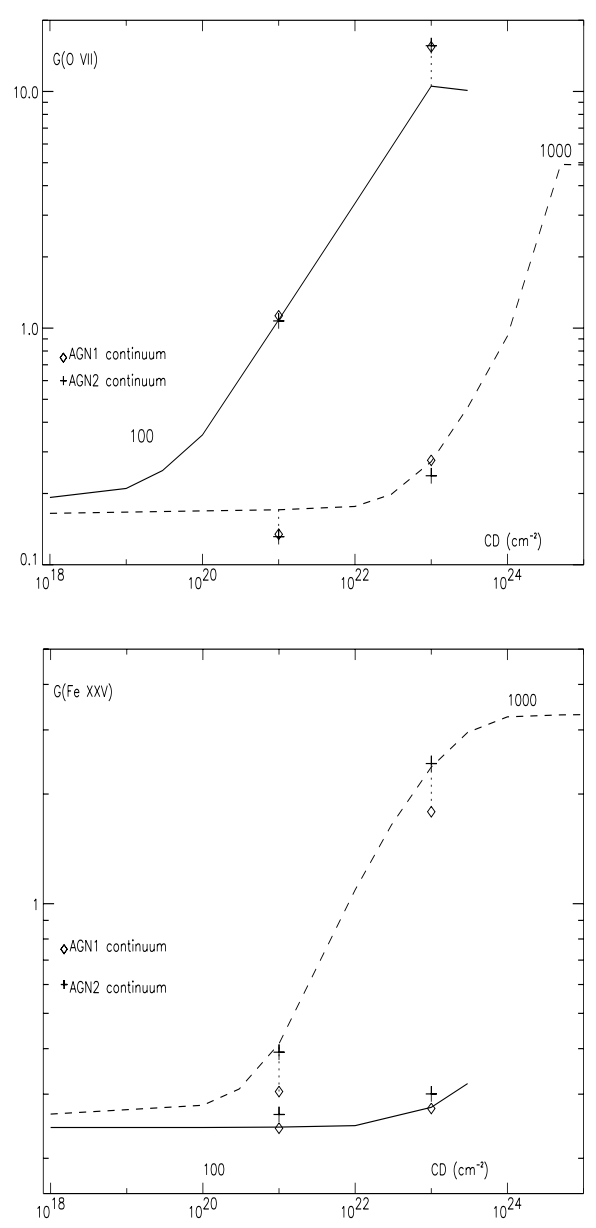

Fig. 10. $G$ ratio in the reflected spectrum versus $C D$ for a medium with $n_{\mathrm{H}}=10^{7} \mathrm{~cm}^{-3}$, photoionized by the AGN1 and AGN2 continua both normalized at $1 \mathrm{keV}$ to the standard continuum. The diamonds and the "+" symbols correspond to repectively the AGN1 and AGN2 continua. The dotted lines on the two figures link models having the same value of $\xi$. The curves are labelled with the value of $\xi$ for the standard continuum.

independent. Thus, if the O VII RRC temperature is derived from the observations, then it is possible to determine a range of the $\xi$-values.

- We show that $R$ does not depend on microturbulence and on the oxygen abundance, except for large column densities and low ionization parameters.

- The $G$ ratios of the He-triplets are very sensitive to the existence of a microturbulent velocity, owing to the decrease of the optical thickness of the resonant lines. This situation can be met in thin media with low $\xi$, and "moderate" and thick media with $\xi \leq 1000$. In these cases, for a given ionization parameter, $G$ decreases when the microturbulent velocity increases. In the other cases, $G$ is independent of the microturbulent velocity, since all He-like lines are optically thin. Thus the column density deduced from the observed $\mathrm{G}$ ratio is larger than that deduced without assuming a microturbulent velocity.

Thus, our study has shown that in modelling X-ray spectra, it is necessary to take into account the various situations met in the

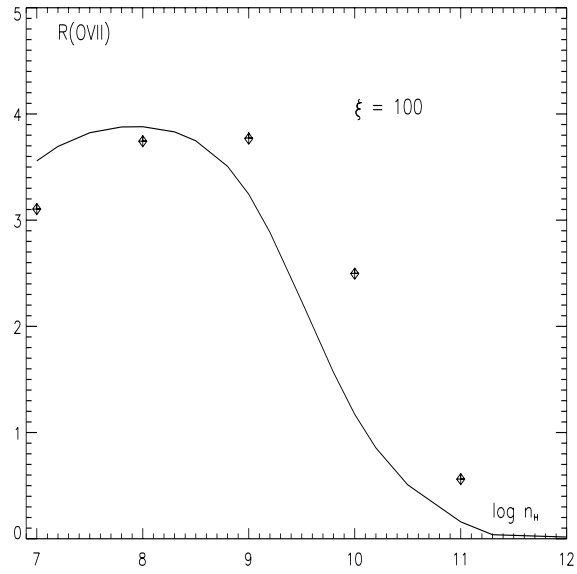

Fig. 11. Same as Fig. 4. The diamonds and the crosses correspond respectively to a model irradiated by the AGN1 and AGN2 continua (see the text for details).

$\mathrm{X}$-ray emission regions of the different photoionized objects, such as Seyfert 1 and Seyfert 2 nuclei, notably the existence of a microturbulent velocity is essential to avoid a misleading interpretation from the He-like diagnostics. The abundance study shows once again the importance of taking into account the interconnection between the different ions in the plasma although we have considered here only the influence of the oxygen abundance.

The present results, computed with improved atomic data, are given with an accuracy of the order of $\pm 10 \%$. It is much better than the accuracy obtained when using the escape probability approximation, as all other photoionization codes do, whatever the sophistication of the atomic model (see Dumont et al. 2003; Collin et al. 2004).

Forthcoming papers will be devoted to building a grid of $\mathrm{X}$-ray spectra taking into account all these possibilities and applying it to the data of the Seyfert 2 galaxies and X-ray binaries.

\section{Appendix: On the relation between dispersion velocity and microturbulence}

The question of turbulent velocity is important, as it can strongly modify the line ratios, but it is not simple. The effect of a turbulent velocity on the line transfer can mimic that of a velocity gradient or a dispersion velocity. However the simple existence of a velocity gradient or a velocity dispersion does not necessarily mean that it intervenes in the line transfer. Let us recall why.

The ionization parameter can be written:

$\xi=\frac{R_{\mathrm{Edd}} L_{\mathrm{Edd}} f_{\mathrm{vol}}}{C D r R_{G}}$

where $R_{\text {Edd }}$ is the bolometric to the Eddington luminosity ratio $L / L_{\mathrm{Edd}}, f_{\mathrm{vol}}$ is the proportion of the line of sight occupied by emitting material $(C D) / R$, i.e. the volumic filling factor, 
and $r$ is the radius of this region expressed in the gravitational radius $R_{G}{ }^{1}$.

First one can show that the medium is not homogeneous in the Seyfert 2 emission region and in the Warm Absorber of Seyfert 1.

Assuming typical values for the Seyfert 2 emission region, one gets:

$\xi \simeq 66\left(R_{\mathrm{Edd}} / 0.1\right) M_{8}^{-1} r_{6}^{-2} n_{5}^{-1}$

where $M_{8}$ is the black hole mass expressed in $10^{8} M_{\odot}$ and $r_{6}$ the radius in $10^{6} R_{G}, n_{5}$ the density in $10^{5} \mathrm{~cm}^{-3}$. It shows that the spectrum would be dominated by heavy He-like emission and not by OVII, unless the density is of the order of or larger than $10^{5} \mathrm{~cm}^{-3}$. For the Warm Absorber of Seyfert 1, the density must be $10^{9} \mathrm{~cm}^{-3}$ or larger.

If $f_{\mathrm{vol}}$ would be equal to unity, one would get

$n \simeq 10^{3} C D_{22} M_{8}^{-1} r_{6}^{-1}$

where $(C D)$ is expressed in $10^{22} \mathrm{~cm}^{-2}$. It is clearly too small, implying that $f_{\text {vol }}$ must be smaller than unity. A similar conclusion can be deduced for the Warm Absorber. One gets for the emission region of Seyfert 2:

$f_{\text {vol }} \simeq 10^{-3}(\xi / 10)\left(R_{\mathrm{Edd}} / 0.1\right)^{-1} C D_{22} r_{6}$.

The emitting region is therefore made of small clouds, whose column density $C D$ can be provided either by one cloud with a column density equal to $C D$, or by a large number of smaller clouds. Since the dispersion velocity inside a cloud is of the order of the thermal velocity, these clouds should have large macroscopic velocities with respect to each others, in order to account for the observed Doppler widths of the emission lines.

Let us consider the case of a unique cloud one a line of sight. The surface coverage factor $f_{\text {surf }}$ is thus:

$f_{\text {surf }} \sim\left(N_{c} R_{\mathrm{c}}^{2}\right) / R^{2}$

where $N_{c}$ and $R_{\mathrm{c}}$ are respectively the number and the radius of the clouds. Since $R_{\mathrm{c}}=(C D) / n$, one gets the maximum number of clouds, which corresponds to a coverage factor equal to unity:

$N_{c}^{\max } \sim 2 \times 10^{4}\left(M_{8} r_{6} n_{5} / C D_{22}\right)^{2}$.

If the number of clouds is smaller than this value, $f_{\text {surf }}$ is smaller than unity. In both cases, a line emitted by one cloud would not be absorbed by another one before escaping the medium. The observed dispersion or gradient velocity would then be due only to the respective motions of the clouds, and would not influence the transfer like microturbulence does. This is the case in the BLR, as proved a long time ago from the study of the line ratios.

Let us consider now the case where the individual size of the clouds is smaller than $R_{\mathrm{c}}=(C D) / n$ and where there are several clouds on a line of sight. Thus there must be $n_{\mathrm{ls}}$ clouds, with $R_{\mathrm{c}}=n_{\mathrm{ls}} R_{\mathrm{c}}^{\prime}$, where $R_{\mathrm{c}}^{\prime}$ is the new radius of the clouds. Thus a line photon emitted by one cloud will cross other clouds before escaping the medium, and it would not be reabsorbed since the other clouds have large velocities corresponding to frequencies in the wings of the line. This is generally treated in the Sobolev approximation. Here, we mimic this case by simply assuming a microturbulent velocity equal to the macroscopic velocity of the clouds with respect to each other, which amounts to assuming that the optical thickness in all lines is so small that, once a photon has been emitted in a cloud, it is never reabsorbed inside the same cloud nor by the others. This means that $n_{\mathrm{ls}}$ should be larger than the optical thickness at the center of the most optically thick lines i.e. $n_{\mathrm{ls}} \geq 10^{5}$. The new surface coverage factor $f_{\text {surf }}^{\prime}$ must be still of the order of unity in order to keep the same line intensities. Since $f_{\text {surf }}^{\prime}$ is equal to $\left(N_{\mathrm{c}}^{\prime} / N_{\mathrm{c}}^{\max }\right) / n_{\mathrm{ls}}^{2}$, where $N_{c}^{\prime}$ is the new number of clouds, we see that $N_{c}^{\prime}$ must be larger than $10^{10} N_{\mathrm{c}}^{\mathrm{max}} \sim 10^{14}$. Since we do not presently know the structure of the emission medium, we have to consider both hypotheses.

\section{References}

Allen, C. W. 1973, Astrophysical quantities (University of London: The Athlone Press)

Collin, S., Dumont, A.-M., \& Godet, O. 2004, A\&A, 419, 877

Coupé, S., Godet, O., Dumont, A.-M., \& Collin, S. 2004, A\&A, 414, 974

Dumont, A.-M., Abrassart, A., \& Collin, S. 2000, A\&A, 357, 823

Dumont, A.-M., Collin, S., Paletou, F., et al. 2003, A\&A, 407, 13

Ferland, G. J., Korista, K. T., Verner, D. A., et al. 1998, PASP, 110, 761

Gabriel, A. H., \& Jordan, C. 1969, MNRAS, 145, 241

Gabriel, A. H., \& Jordan, C. 1972, in Case studies in atomic collision physics, ed. E. W. McDaniel, \& M. R. C. McDowell, 2, 209

Gabriel, A. H., \& Jordan, C. 1973, ApJ, 186, 327

Kallman, T. R., \& Krolik, J. H. 1995, XSTAR, a Spectral Analysis Tool, Users Guide

Kinkhabwala, A., Sako, M., Behar, E., et al. 2002, ApJ, 575, 732

Laor, A., Fiore, F., Elvis, M., Wilkes, B. J., \& McDowell, J. C. 1997, ApJ, 477, 93

Ogle, P. M., Brookings, T., Canizares, C. R., Lee, J. C., \& Marshall, H. L. 2003, A\&A, 402, 849

Porquet, D., \& Dubau, J. 2000, A\&AS, 143, 495

\footnotetext{
${ }^{1}$ We refer to $R_{G}$ instead of a real dimension in parsec, as the locations and sizes of the different regions around a massive black hole are all scaled by the mass of the black hole. For instance, the radius of the BLR and of the Warm Absorber in a Seyfert 1 nucleus is of the order of $10^{4} R_{G}$, and that of the mirror in a Seyfert 2 nucleus is of the order of $10^{6} R_{G}$.
} 